\title{
Researches and Applications of Ant Colony Algorithm
}

\author{
Xiayun $\mathrm{Hu}$ \\ Faculty of Automation \\ Guangdong University of Technology \\ Guangzhou, China \\ Huxiayun666@163.com
}

\author{
Yanfei Zhu \\ Faculty of Automation \\ Guangdong University of Technology \\ Guangzhou, China \\ yanfei_zhu@163.com
}

\begin{abstract}
Ant colony algorithm is a typical algorithm of swarm intelligence algorithm. This article introduces the recent development of this algorithm both in principles and in applications. From the basic principle of ant colony algorithm, the paper evaluates its future development.
\end{abstract}

Keywords-Ant colony algorithm, Swarm intelligence, Flocking, $A C O$

\section{INTRODUCTION}

A cluster can be defined as a group (Generally as a movement) surrogates. They can give mutual communications by actions with their local environments (directly or indirectly). The interactions of these surrogates cause to collectively distribute, then to solve some type questions. The community intelligence has the system quality. Various simple agents in this system inact with the local environments, emerging special behavior to solve problems. The computable group intelligence is the result of this behavior [1]. Swarm intelligence has lots of branches, such as inherit algorithm [2], simulation anneal [3], Beam Search [4], Tabu Search [5, 6], ants algorithm and particle swarmoptimize $[7,8]$ etc.

Ant colony algorithm (ACO) is a typical optimization algorithm of swarm intelligence. It can be used to optimize the paths for searching the possible solutions. It was proposed by Marco Dorigo [9] in his doctoral dissertation in 1992. The inspiration comes from ants searching for food and finding the path of the target.

ACO has some excellent characters such as intelligent search, global optimization, strong robustness, positive feedback, distributed computation and easiness to combine with other algorithm, etc. Its positive feedback can accelerate the evolution process. The good distributed computation not only makes this algorithm easy to be implemented in parallel, but also makes individuals in good information transmission for finding better solutions. ACO combines with a variety of heuristic algorithms easily, then to improve the performance of its own algorithm. The strong robustness improves the basic performance of the ant model, and makes ACO can be used for other problems.

\section{ANT COLONY ALGORITHM For RESEARCH REVIEWED}

Because the TSP problem is the first application of ACO, so the paper uses the TSP problem as an example for better comprehension of ants algorithm process. $m$ is the quantity of ants, $d_{i j}(i=1,2,3,4 \ldots \ldots . n)$ means the distance between the city $i$ and the city $j . b_{i}(t)$ is the quantity of ants located in the city $i$ at the $t$ clock. Therefore, $m=\sum_{i=1}^{n} b_{i}(t) \cdot \tau_{i j}(t)$ means the information content remaining on the $i, j$ line at the $t$ moment. The information content is equal on each path at the start time, namely, $\tau_{i j}(0)=c$. Among them, $c$ is a constant. Ant $k(k=1,2, \ldots, m)$ decides to transfer direction according to information content of each path in the process of exercising. $p_{i j}^{k}(t)$ means the probability which ant $k$ transfers from location $i$ to location $j$ at the $t$ moment

$$
p_{i j}^{k}(t)=\left\{\begin{array}{l}
\frac{\tau_{i j}^{a}(t) \eta_{i j}^{\beta}(t)}{\sum \tau_{i s}^{\alpha}(t) \eta_{i s}^{\beta}(t)}, j \in \text { allowed }_{k}, \text { se allowed } \\
0, \text { otherwise }
\end{array}\right.
$$

Among them, allowed $=\{0,1, \ldots, n-1\}-$ tabu $_{k}$ means the ant $k$ allowes to choose city at next step. $a$ is the heuristic divisor, its value is more big, the ant will more incline toward the selected path passed by other ants, then the cooperation of ant is more strong; $b$ is the expected heuristic factor, its value is more big, the status transition is closer to greedy rule $\eta_{i j}(t)=1 / d_{i j}$. To the ant $k_{\text {speaking, }}$ $d_{i j}$ is more small, $\eta_{i j}(t)$ is more big, $p_{i j}^{k}(t)$ is also more big.

In order to avoid excessive residual pheromone lead to residual information cover heuristic information, each of ants finishes walking one step or finishes walking all cities(A circulating end). It should update the remain information. The update strategy imitates the human brain memory characteristic. Thus, the information on path $(i, j)$ may do adjust according to the following rules at $\mathrm{t}+\mathrm{n}$ moment

$$
\begin{aligned}
& \tau_{i j}(t+n)=(1-\rho) \cdot \tau_{i j}(t)+\rho \cdot \Delta \tau_{i j}(t) \\
& \Delta \tau_{i j}(t)=\sum_{k=1}^{m} \Delta \tau_{i j}^{k}(t)
\end{aligned}
$$


In the type, $\rho$ is usually called pheromone volatile divisor, $1-\rho$ is the information residue coefficient. In order to prevent pheromone infinite accumulation, $\rho$ scope for: $\rho \subset[0,1) ; \Delta \tau_{i j}^{k}(t)$ is the ant $\mathrm{K}$ stays information on path $(i, j)$ in this cycle. $\Delta \tau_{i j}(t)$ is the increment of information on path $(i, j)$ in this cycle.

According to the different of the pheromone refresh strategy, M Dorigo [ 9] put forward three kinds of different basic ant colony algorithm model, Ant-Cycle model, AntQuantity model and Ant - Density model. The difference lies in the method of $\Delta \tau_{i j}^{k}(t)$.

The Ant-Cycle model uses global information, namely, the ant completed a cycle and updated all pheromone on the path. It is perfect in solving TSP, so people usually adopt fomula (4) as the basic model of ant colony algorithm.

Maniezzo and Carbonsro [10, 11] developed approximated nondeterministic tree search(ANTS) . It is an extension of the ant system. ANTS and ant system have the following different: (1)The method of transition probability calculation; (2) The global update rules (3) The method of avoid stagnation. ANTS used the follwing formula(9) calculated transition probability.

$$
p_{i j}^{k}(t)=\left\{\begin{array}{l}
\frac{a \tau_{i j}(t)+(1-a) \eta_{i j}(t)}{\sum a \tau_{i \mu}(t)+(1-a) \eta_{i \mu}(t)}, j \in n_{i}^{k}(t) \\
0, \text { otherwise }
\end{array}\right.
$$

$n_{i}^{k}$ Contains all the available and follow-up node of the current node $i$. Pheromones updated after all ants finished to establish path .Pheromones updated adopted:

$$
\tau_{i j}(t) \leftarrow(1-\rho) \tau_{i j}(t) \text { and } \Delta \tau_{i j}(t)=\sum_{k=1}^{n_{k}} \Delta \tau_{i j}^{k}(t) \quad \text { Pheromone }
$$

burst size $\Delta \tau_{i j}^{k}(t)$ of each ant also avoids premature stagnation: If the ant solution is below the average expenses, reduces the corresponding pheromone strength; Conversely increases the pheromone strength. This algorithm can make the search updating mechanism better in the final stage, And it can avoid excessive development on the initial phase .

Ant colony algorithm has many other structures such as the continuous colony algorithm(CACA) [12,13], Immunized Ant Colony Optimization (IACO)[14], Rapid ant system [15], the maximum minimum ant system [16], the ant ranking system [17].

\section{ANT COLONY AlgORIthM UTILIZES REVIEWED}

ACO algorithm is applied to many optimization problems, most of which are discrete problems. They include secondary allocation [18], job scheduling problems [19], subset problems and other classic problems. practical problems contain network routing, vehicle route planning [20], electric power system of economic scheduling and data mining, biological information. Subset problems are such as traveling salesman problem [21], vehicle route planning problems and all kinds of scheduling problem. ACO algorithm can be used to resolve related public traffic system. Its goal is to maximize direct customer flow. In distribution problems, ACO is used to solve the problems such as circuit design [22], frequency distribution [23] , keyboard arrangement, neural network training schedules, etc. In subset problems, ACO is very useful for constructing k subset of technical tree [24], the biggest independentset [25] and the minimum Stan tree [26], etc. Also, in grouping problems, ACO is important for solving the problems such as the cutting blanking problem [27] and the data clustering problem [28].

\section{A. Network routing - communication problems}

With the wide distribution of the Internet, as well as the increasing demand of multimedia application on quality of service (QoS), various service applications which provide the QoS on the network put forward different requirements. Whereas network routing is the one key to achieve QoS . Ant colony optimization can be used to solve the QoS problems of multicast routing which includes constraint conditions such as broadband, delay, packet traffic and minimum cost $[29,30,31]$. Obviously it is superior to the traditional link-state routing algorithm. Ryan etc [32] used ant colony optimization to solve the WDM optical networks dynamic topology optimum transmission planning problem better. Mohammad etc [33] utilized this method to solve mobile Network (MAN-ET) optimal planning problem.WSN (Wire-less be Wireless) [34] routing is one of the important technology for WSN application, which relates to the stability and strength of whole Network . Ant Colony Optimization (ACO) is a kind of effective method of WSN routing in the routing field. It has remarkable advantages compared with the previous algorithm, such as saving energy to prolong the life cycle of the whole network. In order to solve the problem of joining the new nodes and the failure of the old nodes of WSN, K.Saleem, N.Fisal and S. Hafizah etc [35]utilized the ant autonomy routing algorithms to choose the optimal path considering the level of node energy, the quality of connection, the rate packet loss, and other factors.

\section{B. Problem of Robot path planning}

Robot path planning is to find a satisfying path which can satisfy some specific requirements in obstacle and bounded Spaces from a starting point to the target position without collision. In recent years, many scholars [36,37] have conducted a series of excellent research work on ant colony optimization. In order to solve the problem of robot obstacleavoidance effectively, as well as expand the adaptability to other specific issues, we can get different optimal trajectory by adjusting the obstacle avoidance coefficient in the field of the ant colony optimization. Russell[24], who is a Australian scholar, has designed a smell sensor for robot navigation mechanism, and analyse the robustness of ant colony optimization in this field deeply. A Swiss scholar, Michael, put the ant colony optimization procedures into the miniature robots, which make many miniature robots cooperate harmoniously as ants to complete complex tasks. The 
investigate results have been reported by the famous international publication 《Nature》 [38]. The professor in Jiangxi polytechnic university, Wen Ruchun etc [39], improved the ant colony optimization in labyrinth path planning problem for computer mice to the MRT robots (32bit LPC2318 SCM), which can achieve good effects. When the robots meet obstacles in less than 0.5 second, they can plan new paths. The improved ant colony potimization enhances the obstacle avoidance precision of robot path planning, and speeds up the planning speed precision, as well as meets the practical needs.

\section{SumMarize AND Prospect}

The basic principle of ant colony algorithm is relatively simple but reflects its superiority in many fields. It has many actual applications and has received good effects. However, there are also some deficiencies. Firstly, theoretical studies are relatively few. Secondly, the description for complex problems is not strong enough. Thirdly, the potential of ant colony algorithm in practical applications are not fully exerted.

In the future, In the future, further research should be done in the ant colony algorithm and it will have broad applications. As a kind of intelligent algorithm, ant colony algorithm will appear a bright future.

\section{ACKNOWLEDGEMENTS}

This project is supported by HeSheng ZhuJiang Innovative Foundation of Guangdong University of Technology.

\section{REFERENCES}

[1] Andries P.Engelbrecht Foundations of Computional Swarm Intelligence Chin Hua University press 2010.09.

[2] J.H. Holland .adapatation in Naturl and ArtificialSystem.University of Michigan press 1957

[3] N.Metropolis,A.W.Rosen bluth,M.N.Rosen bluth, a. h. Teller , and E. Teller .Equations of State Calculation by Fast Computing Machines.Journal of Chemical Physic,21: 1087-1092,1958

[4] P.S. Ow and T.E.Morton.Filtered Beam Search inScheduling. International Joural ofProduction Rexearch,26:297-307,1988.

[5] F.Glover.Future Path for Integer Programmingand Artificial Intelligence.Computers and Operations Research,13:533-549,1986.

[6] A.Hertz,E. Taillard,andR.de Werra.A Tutorial on Tabu Search. In Proceedings of Giornate di Lavoro AIRO'95 (Enterprise Systems:Management of Technical and Organizational Changes ),pages 13-24,1995.

[7] R.C.Eberhart and J.Kennedy.A New Optimizer using Particle Swarm Theory.In Proceedings of the Sixth International Symposium on Micromachine andHuman Science,pages 39-43,1995.

[8] J.Kennedy and R.C Eberhart.Particle Swarm Optimization.In Proceedings of the IEEE International JointConference on Neural Networks,pages 1942-1948.IEEE Press, 1995.

[9] M Dorigo , V Maniezzo A,Coloni ColoniA,Dorigo M,ManiezzoV.Distributed optimization by ant colonies[A].Proc 1st European conf Artificial Life[C].Pans,France:Elsevier,1991.134-142

[10] V.Maniezzo and A. Carbonaro Ant Colony Optimization: An Overview.In C. Ribeiro,editor,Essays and Surveys in Metaheuristics,pages21-44.Kluwer,1999
[11] V.Maniezzo and A. Carbonaro.An ANTS Heuristic fou the Frequency Assignment Problem.Future Generation Computer Systems, 16(9):927-935,2000

[12] M.Duran Toksar "An heuristic approach to find the global optimum of function , " Journal of Computational and Applied Mathematics,vol.209,pp.160-166,2007.

[13] J.Dreo and P.Siarry, "An ant colony algorithm aimed at dynamic continuous optimization,"Applied Mathmatics and Computation,vol.181,pp.457-467,2006.

[14] S. H. Pourtakdoust and H. Nobahari, "An Extension of Ant Colony System to Continuous Optimization Problems", In M. Dorigo, M. Birattari, C. Blum, L. M. Gambardella,F. Mondada, and T. Stuetzle, (eds.), Ant Colony Optimization and Swarm Intelligence. Fourth International Workshop, ANTS 2004, LNCS 3172, Springer Verlag, Berlin, Germany,2004, pp. 294-301.

[15] E.D.Taillard and L.M.Gambardella.Adaptive Memories for the Quadratic Assignment Problem Technical report ,IDSIA,Lugano,Switzerland,1997

[16] T.Stvtzle and H. Hoos.MAX-MIN Ant System and Local Search for The TravelingSalesman Problem.In Proceedings of the IEEE International Conferenceon EvolutionaryComputation,pages 309314,1997

[17] B. Bullnheimer, R. Hartl,and C. Strauss. Parallelization Strategies for the Ant System.In G. ToraldoA. Murli, P. Pardalos, editor, Kluwer Series on Applied Optimization,pages 87-100,1997

[18] T.C.Koopmans and M.j. Beckman. Assignment Problems and the Location of Economic Acticities Econometrica,25:53-76,1957

[19] A.Baum,B. Bullnheimer,R.F.Hartl,and C.Strauss.An Ant Colony Optimization Approach for the Single Machine Total Tardiness Problem.In Proceedings of the IEEE Congress on Evolutionary Computation,page 1450,1999

[20] M.Konishi,T.Nishi,k.Nakano.and K.Sotobayashi. Evolutionary Routing Method for Multi Mobile Robors in Transportation.In Proceeding of the IEEE I nternational Sympositium,on Intelligent Control,pages490-495,2002

[21] M.Dorigo.Optimization,Learning and Natural Algorithms,PHD thesis,Politecnico di MILANO,1992

[22] M.Abd-El-BARR,S.M.Sait, and B..A.B.Sarif,Ant Colony Algorithm for Evolutionary Design of Arithmetic Circuits .In Priceedings of the 15th International Conference on Microelectronics,Pages 198201,2003

[23] C.Blum.Ant Colony Optimization for the Edge-Weighted kCardinalty Tree Problem.In Proceeding of the Genetic and Evolutionary Compution Conference,pages27-34,2002

[24] Y. Li and Z. Xu.An Ant Colony Optimization Heuristic for Solving Maximum Independdent Set Problems .In Proceedings of the Fifth International Conference on Computational Intelligence and MultimendiaApplications,pages206-211,2003.

[25] S. Gosavi,S. Das, S. Vaze,G. Singh,andE. Buehler. Obtaining Subtrees from Graphs:An Ant Colony Approach .In Proceedings of the IEEE swarm Intelligence Symposition,pages 160-166,2003

[26] J. Levine and F.Ducatelle.Ant Colony Optimisation and Local Search for Bin Packing and Cutting Stock Problems .Journal of the Operational Research Society,Special Issue on Local Search, 55(7):705-716,2002

[27] C-F. Tsai,C-W.Tsai,H-C. Wu and T.Yang. ACODF: A Nove Data Clustering Approach for Data Mining inLarge Databases.Journal of Systems and software, 73:133-145,2004

[28] Lu G Y, Liu Z QoS multicast routing basedon ant algorithm in internet $[\mathrm{J}]$. The $\mathrm{J}$ of China Universities of Posts and Telecommunication,2000, 7(4):12-17.

[29] Chu C H,Gu J Hou X,et a1.A heuristic ant algorithm for solving OoS muhicast routing problem[A]. Proc of 2002 Congress on EvolutionaryComputation[C]. Honolulu, 20021630-163[26] 
[30] DingJ L, ChenZ Q, Yuan Z Z. Dynamic optimization routing method basedon ant V. adaptive algorithm [J].Control and Decision, 2003, 18(6) : 751-753.

[31] Ryan M G, RichardS B. Dynamic wavelength routing inWDM networks via ant colony optimization[A]. Proc of 3rd IntWorkshop ANTS[C]. Brussels,2002.250-255.

[32] Mohammad T I, ParimalaT, Ruppa KT. A parallel ant colony optimization algorithm for all-pairrouting in MANETs[A]. Proc of the Int Parallel and Dis - thbuted Processing Symposium[C]. Nice, 2003.259-266.

[33] XueHan MA Hong-xu Swarm intelligence based dynamic obstacle avoidance for mobile robots under unknownenvironment using WSN J. Cent. South Univ. Technol. (2008)15: 860-868

[34] K.Saleem, N.Fisal， S.Hafizah， s.kamilahand Rozeha A.Rashid.Ant based Self-organizedRouting protocol for WirelessSensor Network[J].International Journal of Communication Networks and InformationSecuity(IJCNIS)Vol.1 No.2,Auguxt 2009
[35] Israel A W,Michael L,Alfred M B. Distributed covering by antrobots using evaporating traces[J]. IEEE Transon Robotics and Automation, 1999, 15(5):918-933

[36] Hoar R,Penner J,Jacob C.Ant trails-An example for robots to follow[A] . Proc of the 2002 Congress onEvolutionary Computation[C]. Honolulu,2001.1910-1915.

[37] Jin F H ,Hong B R,Gao Q J. Path planning for free-flying space robot using antalgorithm[J]. Robot,2003。24(6) : 526-529

[38] Michael J B K,Jean-Bernard B,LaurentK Ant-like task and recruitment in Cooperative robots[J] Nature,2000,406(31):992—995.

[39] WEN Ru-chun, XU Ying, WANG Zu-lin Study and Application on Maze PathPlaning Based on Improve ant Colony Algorithm .journal of jiangxi university of science and technology Apr.2010 